\title{
Metabolite toxicity determines the pace of molecular evolution within microbial populations
}

\author{
Elin E. Lilja ${ }^{1,2}$ and David R. Johnson ${ }^{1 *}$
}

\begin{abstract}
Background: The production of toxic metabolites has shaped the spatial and temporal arrangement of metabolic processes within microbial cells. While diverse solutions to mitigate metabolite toxicity have evolved, less is known about how evolution itself is affected by metabolite toxicity. We hypothesized that the pace of molecular evolution should increase as metabolite toxicity increases. At least two mechanisms could cause this. First, metabolite toxicity could increase the mutation rate. Second, metabolite toxicity could increase the number of available mutations with large beneficial effects that selection could act upon (e.g., mutations that provide tolerance to toxicity), which consequently would increase the rate at which those mutations increase in frequency.

Results: We tested this hypothesis by experimentally evolving the bacterium Pseudomonas stutzeri under denitrifying conditions. The metabolite nitrite accumulates during denitrification and has pH-dependent toxic effects, which allowed us to evolve P. stutzeri at different magnitudes of nitrite toxicity. We demonstrate that increased nitrite toxicity results in an increased pace of molecular evolution. We further demonstrate that this increase is generally due to an increased number of available mutations with large beneficial effects and not to an increased mutation rate.
\end{abstract}

Conclusions: Our results demonstrate that the production of toxic metabolites can have important impacts on the evolutionary processes of microbial cells. Given the ubiquity of toxic metabolites, they could also have implications for understanding the evolutionary histories of biological organisms.

Keywords: Experimental evolution, Denitrification, Nitrite toxicity, Microbial populations, Molecular evolution

\section{Background}

The production of toxic metabolites is an important factor that shapes the spatial and temporal arrangement of metabolic processes within microbial cells [1]. Toxic metabolites may form as intermediates, side products, or end products of metabolism, and there are numerous examples of how microbial cells arrange metabolic processes in space and time to prevent the accumulation of these metabolites or to divert them away from sensitive biological processes [1]. Consider the denitrification process whereby some types of microbial cells sequentially reduce nitrate $\left(\mathrm{NO}_{3}^{-}\right)$to nitrite $\left(\mathrm{NO}_{2}^{-}\right)$, nitric oxide $(\mathrm{NO})$, nitrous oxide $\left(\mathrm{N}_{2} \mathrm{O}\right)$ and finally to dinitrogen gas

\footnotetext{
* Correspondence: david.johnson@eawag.ch

'Department of Environmental Microbiology, Eawag, Überlandstrasse 133, 8600 Dübendorf, Switzerland

Full list of author information is available at the end of the article
}

$\left(\mathrm{N}_{2}\right)$ to yield energy (Fig. 1) [2]. The metabolic intermediate nitric oxide is a free radical that has cytotoxic effects on cell division and forms metal-nitrosyl complexes with enzymes $[3,4]$. Indeed, nitric oxide performs many of the antimicrobial functions of macrophages [5]. Thus, denitrifying microorganisms must maintain nitric oxide at low concentrations during denitrification. To achieve this, nitric oxide regulates the transcription of denitrification genes [4], suggesting regulatory solutions to control the production and consumption rates of nitric oxide and prevent its accumulation. In addition, denitrifying microorganisms typically produce and consume nitric oxide in the periplasmic space of the cell, thus preventing nitric oxide from interacting with sensitive biological processes that occur within the cytoplasm [6].

While it is clear that the production of toxic metabolites have shaped the spatial and temporal arrangement 


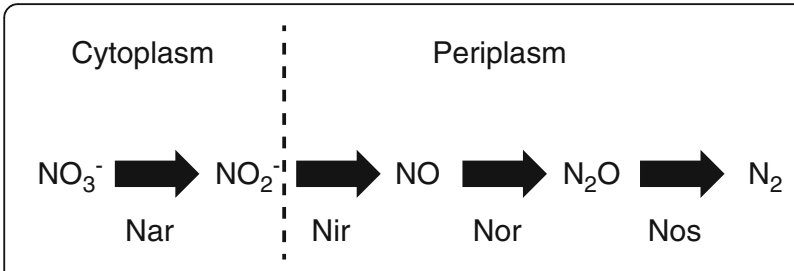

Fig. 1 The denitrification pathway of P. stutzeri. Nitrate is first reduced to nitrite in the cytoplasm. Nitrite is then actively transported to the periplasmic space where it is further reduced through several intermediates to dinitrogen gas. The consequence is that nitrite accumulates in the periplasm. Enzymes: Nar, nitrate reductase; Nir, nitrite reductase; Nor, nitric oxide reductase; Nos, nitrous oxide reductase

of metabolic processes within microbial cells, it is less clear how metabolite toxicity affects the pace of molecular evolution itself. In this context, we use the term "pace of molecular evolution" to refer to the number of mutations that accumulate per generation and not to the number of mutations that accumulate per unit time. Metabolite toxicity could affect the pace of molecular evolution by at least two distinct mechanisms. First, metabolite toxicity might directly (e.g., acting on DNA) or indirectly (e.g., via general stress response) increase the mutation rate, thus providing a larger set of mutations for selection or drift to act upon [7, 8]. Second, metabolite toxicity might decrease the absolute fitness of the cell; that is, metabolite toxicity might cause slower growth rates or lower yields due to their potentially deleterious effects on biological processes. This could result in increased selection pressure and increased numbers of available mutations with large beneficial effects for selection to act upon (e.g., mutations that provide tolerance to or eliminate metabolite toxicity), thus increasing the rate at which those mutations increase in frequency $[9,10]$.

Our main objectives were to experimentally test whether the toxicity of a single metabolite does indeed increase the pace of molecular evolution and to determine by which mechanisms such an effect might emerge (i.e., by increasing the mutation rate or by increasing the number of available mutations with large beneficial effects for selection to act upon). To address these objectives, we developed an experimental system where we could manipulate the toxicity of a single metabolite. We then experimentally evolved replicated populations of bacteria at two distinct levels of metabolite toxicity, quantified the types and numbers of mutations that accumulated during experimental evolution, and tested whether metabolite toxicity affects the pace of molecular evolution.

Our experimental system is based on the denitrifying bacterium Pseudomonas stutzeri A1501 (hereafter referred to as $P$. stutzeri), which is a facultative anaerobe with a fully sequenced genome $[11,12]$. In the absence of oxygen, $P$. stutzeri can use nitrogen oxides as terminal electron acceptors to support its growth [13]. P. stutzeri sequentially reduces nitrate $\left(\mathrm{NO}_{3}^{-}\right)$to nitrite $\left(\mathrm{NO}_{2}^{-}\right)$, nitric oxide $(\mathrm{NO})$, nitrous oxide $\left(\mathrm{N}_{2} \mathrm{O}\right)$, and finally to dinitrogen gas $\left(\mathrm{N}_{2}\right)$ using different enzyme complexes for each reduction step (Fig. 1) [6]. An important feature of this experimental system is that the metabolite nitrite accumulates in batch culture and has $\mathrm{pH}$-dependent toxic effects $[14,15]$. As the $\mathrm{pH}$ decreases, nitrite increasingly generates nitrous acid $\left(\mathrm{HNO}_{2}\right)$, which uncouples proton translocation [16, 17]. In addition, nitrite increasingly and spontaneously generates nitric oxide radicals that impose cytotoxic effects on cell division and form metal-nitrosyl complexes with enzymes [3]. The consequence is that, as the $\mathrm{pH}$ decreases, the increased toxicity of nitrite has negative effects on growth and metabolic activity $[15,18]$. In general, nitrite toxicity has negligible effects at $\mathrm{pH} 7.5$ and severe effects at $\mathrm{pH} 6.5$, while $\mathrm{pH}$ itself has no measureable effects under the same $\mathrm{pH}$ range [15]. The $\mathrm{pH}$ of the culture medium can therefore be used to manipulate nitrite toxicity without creating substantial confounding factors [15], thus allowing us to test the hypothesis that increased nitrite toxicity increases the pace of molecular evolution. Indeed, we previously demonstrated that $\mathrm{pH}$ itself has no statistically significant effects on the growth of $P$. stutzeri under the experimental conditions used in this study while nitrite is non-toxic at $\mathrm{pH} 7.5$ but severely toxic at $\mathrm{pH} 6.5$ [15].

\section{Results}

Increased nitrite toxicity accelerates molecular evolution Our first objective was to experimentally test whether increased toxicity of a single metabolite, nitrite $\left(\mathrm{NO}_{2}^{-}\right)$, increases the pace of molecular evolution. To achieve this objective, we experimentally evolved eight populations of $P$. stutzeri at $\mathrm{pH} 6.5$ (strong nitrite toxicity) and eight populations at $\mathrm{pH} 7.5$ (weak nitrite toxicity) for approximately 700 generations. We then randomly selected a single clone from each evolved population, sequenced its genome, and quantified the number of mutations within that clone when compared to the ancestral clone (Additional file 1: Table S1-2). We found that more mutations accumulated in clones evolved at $\mathrm{pH} 6.5$ than at $\mathrm{pH} 7.5$, thus supporting our main hypothesis (Wilcoxon rank-sum test; Bonferroni-corrected $P<0.01, \mathrm{n}_{1}=\mathrm{n}_{2}=8$ ) (Fig. 2). We further found that one clone evolved at $\mathrm{pH} 6.5$ accumulated five times more mutations than the other clones evolved at $\mathrm{pH}$ 6.5. This clone contains a loss of function mutation in the $u v r A$ gene, which encodes for subunit A of excinuclease, a protein involved in nucleotide excision-based DNA repair [19, 20]. Thus, this mutation likely caused an 


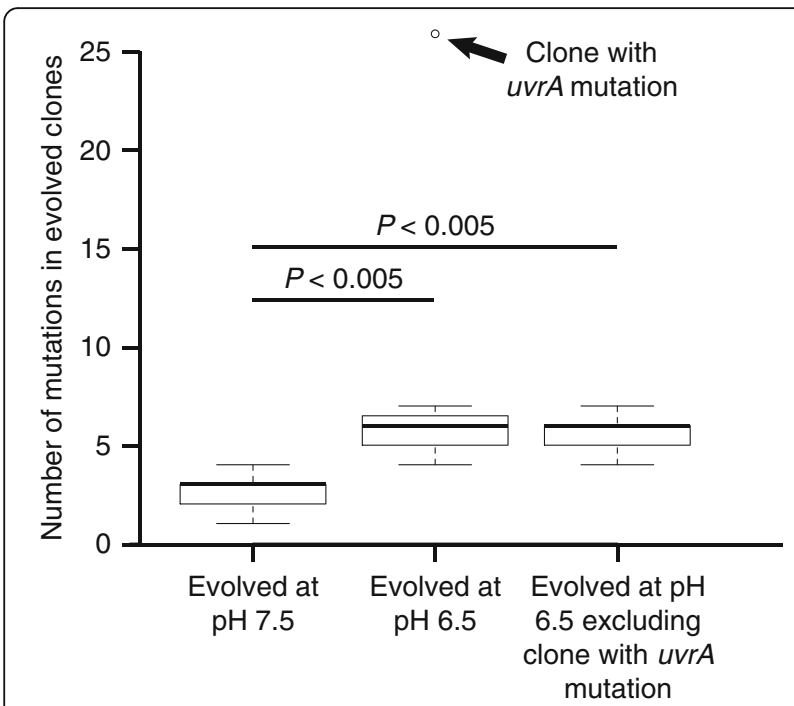

Fig. 2 The number of mutations that accumulated in clones after evolution at pH 6.5 (strong nitrite toxicity) or pH 7.5 (weak nitrite toxicity). Horizontal bars and $P$-values indicate the outcomes of two-sample Wilcoxon rank-sum tests. The arrow indicates the clone with a mutation in uvrA. Data are presented as Tukey box-plots

increased mutation rate in this clone. We therefore further tested whether more mutations accumulated in clones evolved at $\mathrm{pH} 6.5$ than at $\mathrm{pH} 7.5$ when this clone was removed from the statistical analysis, and this was indeed the case (Wilcoxon rank-sum test; Bonferronicorrected $P<0.01, \mathrm{n}_{1}=8, \mathrm{n}_{2}=7$ ) (Fig. 2). This was also true when we only took into account non-synonymous mutations within protein coding regions (Additional file 1: Figure S1). Thus, our data supports the hypothesis that the increased reactivity of a single metabolite can increase the pace of molecular evolution.

Increased mutation rates are unlikely (but not unequivocally excluded) to account for the increased pace of molecular evolution. The increased numbers of mutations in clones evolved at $\mathrm{pH} 6.5$ (strong nitrite toxicity) could be due to either increased mutation rates or increased numbers of available mutations with large beneficial effects that selection could act upon. They could also be caused by epistatic effects among mutations or by demographic differences between treatments. We investigated whether increased mutation rates were important by categorizing all mutations by mutation type: point mutations (non-synonymous, synonymous or intergenic), indels (genic and intergenic), large deletions (>250 bp), mutations conferred by insertion sequences, and mutations in ribosomal genes (Fig. 3). We reasoned that, if the accelerated pace of molecular evolution was predominantly caused by increased mutation rates, than we would expect increased numbers of synonymous substitutions to accumulate within those clones. This was not the case. There was no significant difference in the

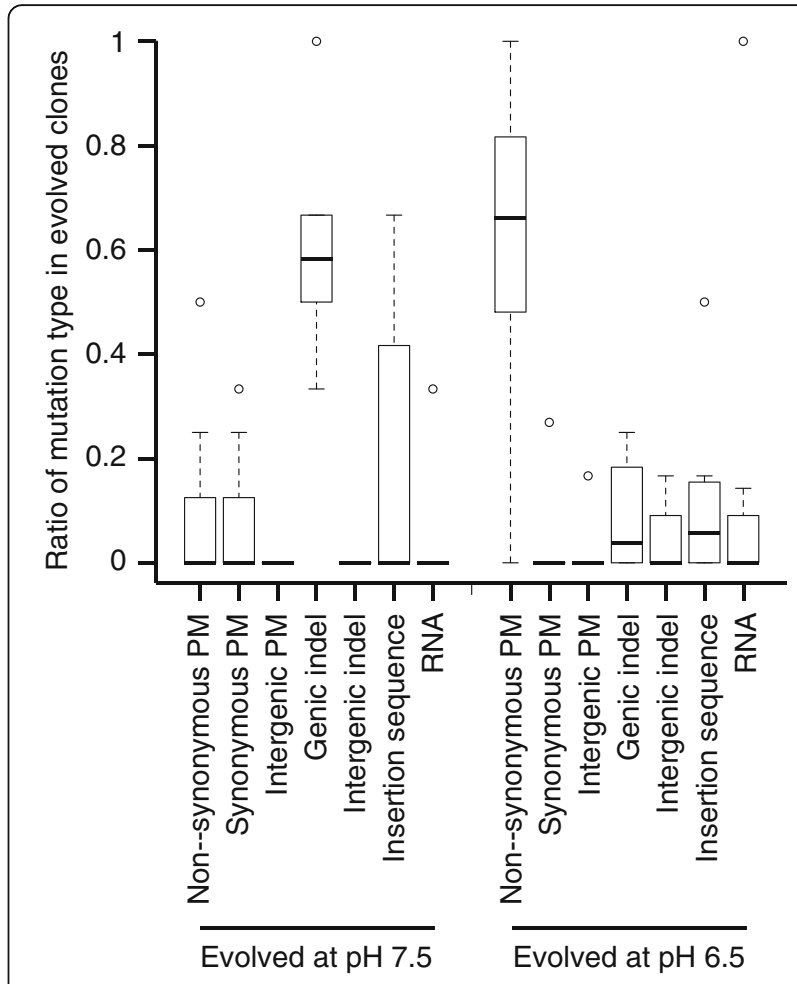

Fig. 3 The types of mutations that accumulated in clones after evolution at pH 6.5 (strong nitrite toxicity) or pH 7.5 (weak nitrite toxicity). Each mutation was categorized by type and frequency among all mutations. Data are presented as Tukey box-plots

frequency of synonymous substitutions (Wilcoxon ranksum test, $P>0.5, \mathrm{n}_{1}=\mathrm{n}_{2}=8$ ) or the absolute abundance of synonymous substitutions (Wilcoxon rank-sum test, $P>0.6, \mathrm{n}_{1}=\mathrm{n}_{2}=8$ ) for clones evolved at $\mathrm{pH} 6.5$ compared to clones evolved at $\mathrm{pH} 7.5$ (Fig. 3). This was true regardless of whether we included or removed the clone with the mutation in the $u v r A$ gene from the analysis. Indeed, we did not detect any synonymous mutations at all in the clones evolved at $\mathrm{pH}$ 6.5, except within the clone that has a mutation within the $u v r A$ gene. Taken together, with the exception of the single clone with a mutation in the $u v r A$ gene, we have no evidence that differences in mutation rates generally explain the increased numbers of mutations that accumulated within clones evolved at $\mathrm{pH} 6.5$ (strong nitrite toxicity). However, we acknowledge that we do not have direct measures of mutation rates, and we instead rely on proxy measures such as comparing rates of synonymous substitutions. It is possible that the absence of different numbers of synonymous substitutions may be due to the low numbers of total mutations that were observed in this study, which could be addressed in the future via sequencing additional clones or investigating longer evolutionary time-scales. 
Interestingly, we did find that clones evolved at $\mathrm{pH} 6.5$ (strong nitrite toxicity) accumulated different types of mutations than those evolved at $\mathrm{pH} 7.5$ (weak nitrite toxicity). Clones evolved at $\mathrm{pH}$ 6.5, which exhibited the increased pace of molecular evolution, accumulated significantly more non-synonymous substitutions than other types of mutations (ANOVA followed by Tukey's HSD post hoc analysis; $P<0.01$ ) (Fig. 3). In contrast, clones evolved at $\mathrm{pH} 7.5$ accumulated significantly more short indels in coding regions than other types of mutations (ANOVA followed by Tukey's HSD post hoc analysis; $P<0.01$ ) (Fig. 3). The different types of mutations did not always occur in different target genes. For example, mutations occurred in the oprQ gene at both $\mathrm{pH}$ conditions, but the mutations are short deletions at $\mathrm{pH} 7.5$ and primarily single-base substitutions at $\mathrm{pH} 6.5$.

\section{Increased nitrite toxicity leads to larger increases in absolute fitness}

If the increased numbers of mutations in clones evolved at $\mathrm{pH} 6.5$ (strong nitrite toxicity) were generally due to increased numbers of available mutations with large beneficial effects that selection could act upon, then we would expect the populations evolved at $\mathrm{pH} 6.5$ to have larger increases in fitness than the populations evolved at $\mathrm{pH} 7.5$ relative to the ancestral clones. We tested this expectation using growth assays and measuring the time for populations to reach stationary phase, which is equivalent to the time required for complete substrate consumption under our experimental conditions [15]. We calculated the relative time for complete substrate consumption as the time for the evolved populations to enter stationary phase $\left(t_{\text {evol }}\right)$ divided by the time for the ancestral populations to enter stationary phase $\left(t_{\mathrm{anc}}\right)$. We report the ratios of $t_{\text {evol }}$ to $t_{a n c}$ in Fig. 4. We found that the populations evolved at $\mathrm{pH} 6.5$ had significantly smaller ratios of $t_{\text {evol }}$ to $t_{\text {anc }}$ than those evolved at $\mathrm{pH} 7.5$ (Wilcoxon rank-sum test; $P<0.001, \mathrm{n}_{1}=\mathrm{n}_{2}=$ 8) (Fig. 4). Overall, the populations evolved at $\mathrm{pH} 6.5$ required approximately $50 \%$ of the ancestral time to reach stationary phase while the populations evolved at $\mathrm{pH} 7.5$ required about $80 \%$ of the ancestral time (Fig. 4). We note that the clone with the mutation in the $u v r C$ gene is not an outlier for these analyses, suggesting that the increased number of mutations resulting from a potentially increased mutation rate does not cause a measurable change in the fitness of this clone. Together, our results are consistent with the increased numbers of mutations that accumulated in populations evolved at $\mathrm{pH} 6.5$ (strong nitrite toxicity) being caused by increased numbers of available mutations with large beneficial effects.

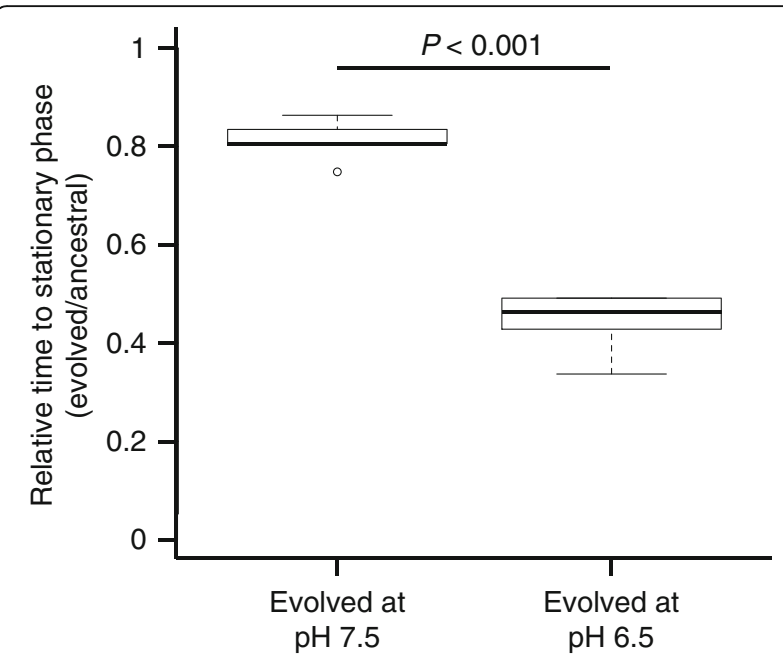

Fig. 4 Relative time to stationary phase for each experimental evolution condition. Data are the time to reach stationary phase for the evolved clones divided by the time to stationary phase for the ancestral clones. The horizontal bar and $P$-value indicates the outcome of a two-sample Wilcoxon rank-sum test. The asterisk indicates $P<0.05$. The data are presented as Tukey box-plots

\section{Increased nitrite toxicity leads to larger increases in competitive fitness}

Because evolution at pH 6.5 (high nitrite toxicity) led to both increased numbers of mutations and larger increases in fitness, we further investigated whether evolution at $\mathrm{pH} 6.5$ also results in increased competitive fitness. We define competitive fitness as the relative fitness of an evolved clone when competed directly against its ancestor in co-culture. To address this, we performed four different competition assays where we competed evolved clones against ancestral clones. We initiated the competition assays with $5 \%$ of evolved cells and $95 \%$ of ancestral cells, as our previous competition assays using an initial ratio of $50 \%$ of evolved cells and $50 \%$ of ancestral cells did not yield any significant differences (Additional file 1: Figure S2). This was likely because the evolved cells accumulated less nitrite. Thus, when they are present at high frequencies, they reduce nitrite accumulation and "rescue" the ancestral cells.

We first tested whether the clones evolved at $\mathrm{pH} 6.5$ (strong nitrite toxicity) improved in competitive fitness more than the clones evolved at $\mathrm{pH} 7.5$ (weak nitrite toxicity), which would be expected given that the clones evolved at pH 6.5 accumulated more mutations and consumed substrates more rapidly as compared to the ancestor (i.e., they required less time to reach stationary phase). To accomplish this, we compared the competitive fitness of the evolved clones when they were competed against the ancestor at the same conditions as they were evolved. We found that the clones evolved at $\mathrm{pH} 6.5$ increased in competitive fitness significantly 
more than the clones evolved at $\mathrm{pH} 7.5$ (ANOVA with a Tukey's HSD post hoc analysis, $P<0.01$ ) (Fig. 5; first boxplot compared to the third boxplot), thus supporting our expectation.

We next assessed competitive fitness in the nonselected environments. First, we compared the competitive fitness of clones evolved at $\mathrm{pH} 7.5$ (weak nitrite toxicity) when competed against the ancestor at $\mathrm{pH} 6.5$ (strong nitrite toxicity) or $\mathrm{pH} 7.5$. The objective was to investigate if the fitness benefits acquired during evolution at $\mathrm{pH} 7.5$ become more pronounced at $\mathrm{pH} 6.5$. We found that the clones evolved at $\mathrm{pH} 7.5$ increased in competitive fitness significantly more when competed at $\mathrm{pH} 6.5$ than at $\mathrm{pH} 7.5$ (ANOVA with a Tukey's HSD post hoc analysis, $P<0.01$ ) (Fig. 5; last boxplot compared to the third boxplot). This indicates that the mutations that accumulated in clones evolved at $\mathrm{pH} 7.5$ have larger benefits at $\mathrm{pH} 6.5$ than at $\mathrm{pH} 7.5$. This could occur, for example, if mutations emerged that reduce the intracellular accumulation of nitrite. While potentially beneficial regardless of the $\mathrm{pH}$, the beneficial effects would be greater at $\mathrm{pH} 6.5$ when nitrite is toxic. Further experiments would be needed to test this possibility.

Next, we compared the competitive fitness of clones evolved at $\mathrm{pH} 6.5$ (strong nitrite toxicity) or $\mathrm{pH} 7.5$ (weak nitrite toxicity) against the ancestor at $\mathrm{pH} 6.5$. The objective was to investigate if evolution at $\mathrm{pH} 6.5$

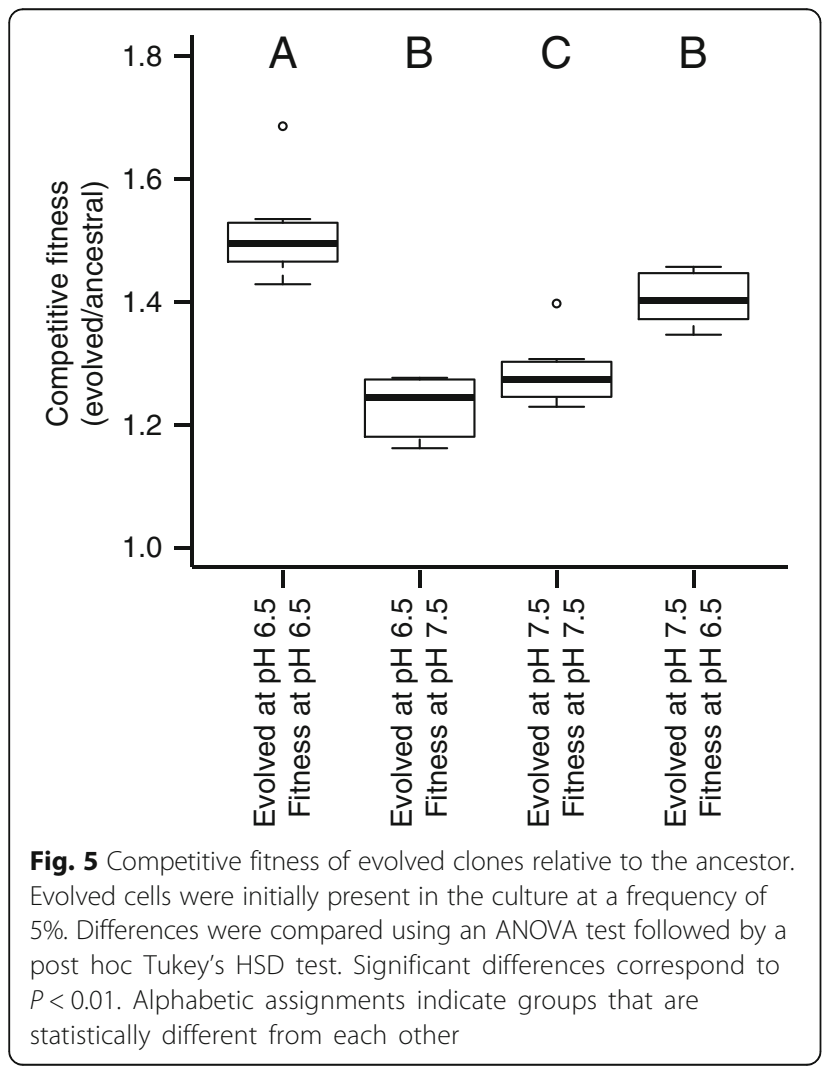

leads to larger fitness benefits at $\mathrm{pH} 6.5$ than evolution at $\mathrm{pH}$ 7.5. Indeed, we found that the clones evolved at $\mathrm{pH} 6.5$ increased in competitive fitness significantly more than the clones evolved at $\mathrm{pH} 7.5$ (ANOVA with a Tukey's HSD post hoc analysis, $P<0.01$ ) (Fig. 5; first boxplot compared to the last boxplot). This indicates that, even though the mutations accumulated in clones evolved at $\mathrm{pH} 7.5$ had larger benefits at $\mathrm{pH} 6.5$ than at $\mathrm{pH} 7.5$, this is not sufficient to account for all of the increased fitness of clones at $\mathrm{pH} 6.5$.

Finally, we compared the competitive fitness of the clones evolved at $\mathrm{pH} 6.5$ (strong nitrite toxicity) or pH 7.5 (weak nitrite toxicity) when competed against the ancestor at $\mathrm{pH} 7.5$. The objective was to investigate if the increased fitness benefits in clones evolved at $\mathrm{pH} 6.5$ have any significant effect at $\mathrm{pH}$ 7.5. We found that there is no significant difference in the competitive fitness between the two evolutionary conditions when assayed at pH 7.5 (ANOVA with a Tukey's HSD post hoc analysis, $P>0.01$ ) (Fig. 5; second boxplot compared to the third boxplot). These results indicate that there are indeed mutations that have a beneficial effect on fitness at $\mathrm{pH} 6.5$ that have no effect at $\mathrm{pH}$ 7.5. Taken together with the outcomes from the other competition assays, our data supports the idea that the larger increase in competitive fitness found after evolution at $\mathrm{pH} 6.5$ is both due to an increased benefit of the same type of mutations that are found at $\mathrm{pH} 7.5$ as well as additional types of mutations that have a benefit only at $\mathrm{pH}$ 6.5. We acknowledge, however, that interactions between mutations may also be critical in addition to individual mutation effects.

\section{Increased nitrite toxicity selects for mutations in genes with a broader variety of functional annotations}

We next tested whether evolution at $\mathrm{pH} 6.5$ (strong nitrite toxicity) not only promotes more mutations to accumulate, but also affects the functional composition of those mutations. To address this, we functionally categorized each gene with a mutation according to existing annotations $[11,21]$. In this analysis, we only included non-synonymous substitutions in coding regions or point mutations in intergenic regions that are likely to be involved in transcription (Fig. 6). Clones evolved at $\mathrm{pH} 7.5$ only have mutations in three functional categories: cell motility, signal transduction and unknown functions. Mutations in these types of functions are also commonly found in clones evolved at $\mathrm{pH}$ 6.5. The numbers of mutations in the clones do not differ between the two conditions (strong or weak nitrite toxicity) for these functional categories. Clones evolved at $\mathrm{pH} 6.5$ had mutations in all of the same categories discussed above, but also have mutations in additional categories: carbohydrate transport and metabolism, lipid transport 


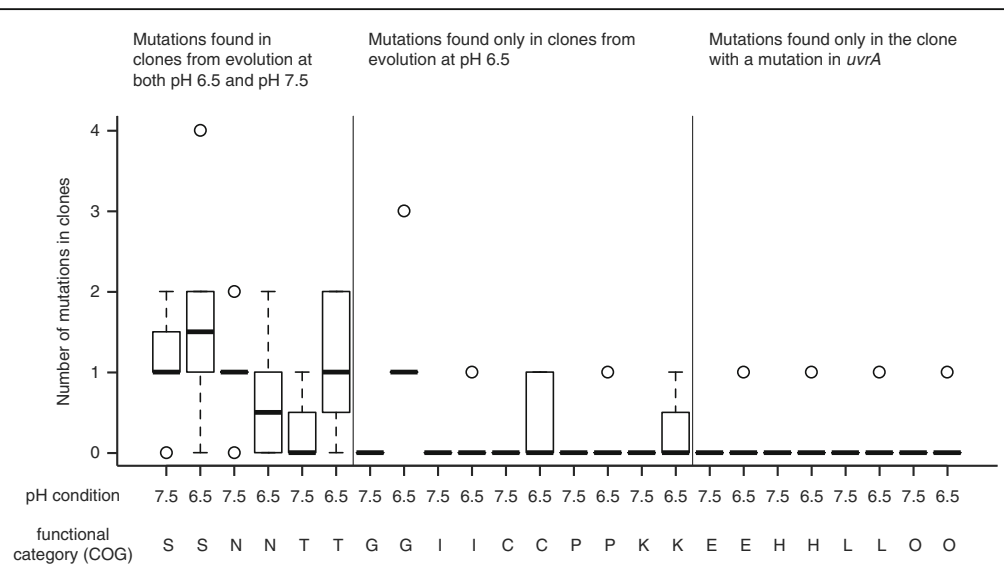

Fig. 6 Functional categorization of genes that contain mutations in evolved clones for each experimental evolution condition. Each mutation was categorized by type. Data are presented as Tukey box-plots. Functional categories (COG): S, function unknown; N, cell motility; T, signal transduction; G, carbohydrate transport and metabolism; l, lipid transport and metabolism; C, energy production and conversion; $P$, inorganic ion transport and metabolism; K, transcription; E, amino acid transport and metabolism; H, coenzyme transport and metabolism; L, replication, recombination and repair; $\mathrm{O}$, posttranslational modification, protein turnover, and chaperones

and metabolism, energy production and conversion, inorganic ion transport and metabolism and transcription. However, the only functional category where this is statistically significant from the clones evolved at $\mathrm{pH} 7.5$ is carbohydrate transport and metabolism (Wilcoxon rank sum test, $\left.\mathrm{n}_{1}=\mathrm{n}_{2}=8, P<0.001\right)$. Indeed, each of the clones evolved at $\mathrm{pH} 6.5$ have a mutation in at least one gene encoding for an enzyme involved in carbohydrate metabolism, while none of the clones evolved at $\mathrm{pH} 7.5$ have a mutation in a gene involved in carbohydrate metabolism. The other functional categories are those where mutations were only found in the clone with a mutation in uvrA (Fig. 6). A few clones evolved at $\mathrm{pH} 6.5$ also have mutations in genes involved in denitrification that were not observed in clones evolved at $\mathrm{pH} 7.5$ (denitrification-related genes are distributed among a variety of functional categories). In conclusion, evolution at $\mathrm{pH} 6.5$ (strong nitrite toxicity) led not only to the accumulation of more mutations but also to the accumulation of mutations with increased diversity of functional annotations.

\section{Populations evolved at increased nitrite reactivity have decreased efficiency in the use of alternative carbon substrates}

We finally tested whether the increased numbers of mutations in populations evolved at $\mathrm{pH} 6.5$ (strong nitrite toxicity) correlates with increased pleiotropic effects under non-selected environments. To accomplish this, we measured the performance of the ancestral and all of the evolved populations when growing on a variety of alternative carbon sources using Biolog PM1 plates. These plates contain 95 individual carbon sources and can be used to measure growth over time. We measured performance as the area under the growth curve $\left(\mathrm{OD}_{600}\right.$ vs time) for each carbon substrate as described elsewhere [22]. We only took into account those carbon sources for which there was visible growth.

First, we compared the populations evolved at $\mathrm{pH} 6.5$ (strong nitrite toxicity) with the ancestor grown at $\mathrm{pH} 6.5$ and the populations evolved at $\mathrm{pH} 7.5$ (weak nitrite toxicity) with the ancestor grown at $\mathrm{pH} 7.5$ (Fig. 7a-b). We found that populations evolved at $\mathrm{pH} 7.5$ had no significant overall change in their ability to use alternative carbon substrates when compared to the ancestor; the average of evolved area $/$ ancestral $_{\text {area }}$ (area = area under curve) does not deviate from one (Fig. 7a; one sample Wilcoxon rank-sum test; $P>0.2$ ). In contrast, populations evolved at $\mathrm{pH} 6.5$ had an overall decrease in carbon source utilization; the average of evolved $_{\text {area }} /$ ancestral $_{\text {area }}$ is significantly lower than one (Fig. 7b; one-sample Wilcoxon rank -sum test; $P<0.05$ ). We also calculated the differences between the two evolution conditions for both the ancestors and the evolved populations; that is, we calculated the average at $\mathrm{pH} 6.5$ and divided it by the average at $\mathrm{pH} 7.5$ for each carbon source for the ancestors and for the evolved populations (Fig. 7c-d). The average of $\mathrm{pH} 6.5_{\text {area }} / \mathrm{pH} 7.5_{\text {area }}$ does not deviate from one for the ancestors, indicating that $\mathrm{pH}$ itself has no effect (Fig. 7c; one-sample Wilcoxon signedrank test, $P>0.3$ ). In contrast, the average of $\mathrm{pH} 6.5_{\text {area }} /$ $\mathrm{pH} 7.5_{\text {area }}$ is significantly lower than one for the evolved populations, indicating that evolution at $\mathrm{pH} 6.5$ does indeed have negative consequences on the utilization of non-selected substrates (Fig. 7d; one-sample Wilcoxon signed-rank test, $P<0.00001)$. Taken together, our data support the conclusion that the increased accumulation of mutations at $\mathrm{pH} 6.5$ led to increased antagonistic 

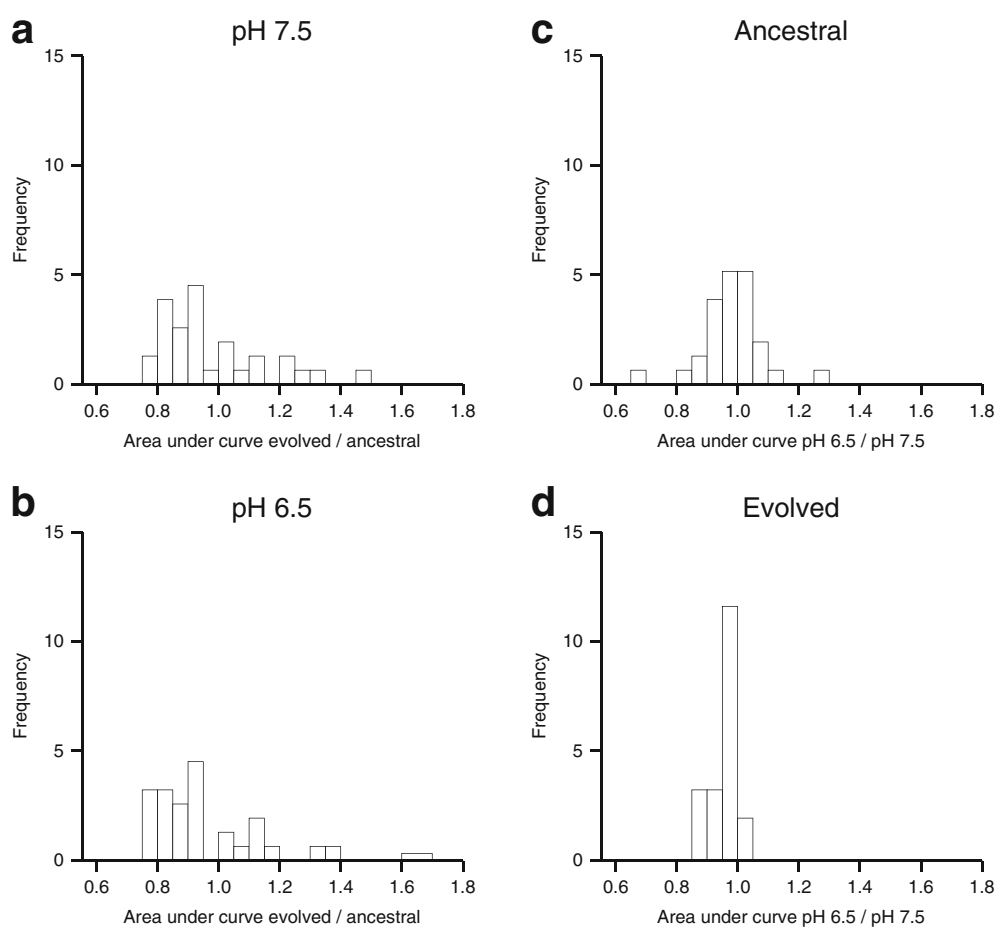

Fig. 7 Performance of evolved and ancestral clones to utilize alternative carbon substrates. Carbon utilization was measured for 95 different carbon substrates. Performance was quantified as the area under the growth curve for each individual carbon substrate $\left(\mathrm{OD}_{600}\right.$ vs. time), which takes into account both growth rate and yield. Data are presented as histograms. (a) Comparison of clones evolved at pH 7.5 with ancestral populations at pH 7.5; (b) comparison of clones evolved at pH 6.5 with ancestral clones at pH 6.5; (c) comparison of ancestral clones at pH 6.5 with ancestral clones at pH 7.5; (d) comparison of clones evolved at $\mathrm{pH} 6.5$ with clones evolved at $\mathrm{pH} 7.5$

pleiotropic effects in non-selected environments. This is likely due to the increased accumulation of mutations in carbohydrate utilization and transport genes at $\mathrm{pH} 6.5$, which may have negative effects on the ability of those evolved populations to utilize alternative carbon substrates.

\section{Discussion}

Our results are consistent with the hypothesis that increased toxicity of a single metabolic intermediate can accelerate the pace of molecular evolution, and this leads to larger increases in both absolute and relative fitness. We conclude that the increased rate of evolution is most likely due to the increased availability of mutations with large beneficial effects that selection can act upon rather than an increase in the mutation rate in response to stressful conditions, the latter of which has been repeatedly observed $[7,8,23,24]$. This is based on evidence that there is no increase in synonymous mutations at strong nitrite toxicity (Fig. 3) and further supported by the fact that the additional mutations that accumulated during evolution at strong nitrite toxicity do not confer a significant additional increase in fitness at weak nitrite reactivity (Fig. 5). Furthermore, while we identified a single clone with five-fold more mutations than the other clones (Fig. 2), this clone did not show improved fitness when compared to the other clones. We note here that differences in $\mathrm{pH}$ may have also contributed towards the different outcomes, but we believe this is unlikely given that differences in $\mathrm{pH}$ over the experimentally evolved conditions have no observable effect on the growth of P. stutzeri [15].

The larger increases in fitness observed after evolution at strong nitrite toxicity were due to two mechanisms. The first mechanism is that the fitness benefits of mutations in genes encoding for certain functions had larger beneficial effects as nitrite toxicity increased. We base this conclusion on the fact that clones evolved at weak nitrite toxicity share mutations in genes with similar functions with clones evolved at strong nitrite toxicity, including mutations in genes involved in cell motility (e.g. flgD, fleQ), signal transduction (phoP), and in genes encoding for membrane proteins (oprE3, PST_2380 [a porin]) (Additional file 1: Table S1-2). This suggests that there are adaptations common to both conditions. Yet, the clones evolved at weak nitrite toxicity have larger increases in competitive fitness when grown at high nitrite toxicity than at low nitrite toxicity (Fig. 5), suggesting that the adaptations common to both conditions have larger benefits at stronger nitrite toxicity. This idea that the benefits of mutations 
can be larger (and thus requiring fewer generations to accumulate within the population) in a less fit background is consistent with several previous studies. For example, a recent study with yeast demonstrated that beneficial mutations have larger benefits in more stressful backgrounds, where the initial fitness was determined by the genotype rather the environment [25]. As another example, mutations conferring antibiotic resistance have larger benefits at higher antibiotic concentrations [26]. Thus, in the case of nitrite toxicity, mutations in certain functions have beneficial effects at both weak and strong nitrite toxicity, but those benefits increase as toxicity increases.

The second mechanism is that mutations emerged that are only beneficial at strong nitrite toxicity. That is, populations evolved at strong nitrite toxicity have larger increases in competitive fitness than populations evolved at weak nitrite toxicity when competed at strong nitrite toxicity (Fig. 5). Additionally, populations evolved at strong nitrite toxicity have no significant difference in the increase of competitive fitness compared to populations evolved at weak nitrite toxicity when competed at weak nitrite toxicity (Fig. 5). This idea is further supported by the fact that mutations in genes encoding for proteins involved in carbon metabolism ( $P y k A, F b p$, Gap-2) and in denitrification were only identified in clones evolved at strong nitrite toxicity (Additional file 1: Table S1-2). These results indicate that mutations in functions such as carbon metabolism and denitrification only have beneficial effects at strong nitrite toxicity.

Our data additionally demonstrates that increased nitrite toxicity not only accelerates molecular evolution, but also reduces the niche breadth of the cells. We found that the increase in the number of mutations during evolution at strong nitrite toxicity led to increased antagonistic pleiotropic effects in non-selected environments, as demonstrated by the decrease in utilization efficiency of alternative carbon sources (Fig. 7). While statistically significant, we caution that these results may not be biologically significant, as the effects are typically small for many of the alternative carbon sources. However, the fact that all lineages evolved at strong nitrite toxicity have mutations in important enzymes involved in glycolysis and gluconeogenesis supports the idea that carbon utilization would be affected in these lineages. This suggests that evolution at increased nitrite toxicity leads to increased metabolic specialization and decreased niche breadth. This decrease in niche breadth may not be unexpected, as there is some evidence supporting the idea that adaptation to more stressful environments leads to increased trade-offs in nonselected environments [27]. Given the large numbers of substrates and intermediates that are present in the natural environment, metabolite toxicity may therefore be an important factor that limits niche breadth and, in turn, promotes biodiversity and the coexistence of different metabolically specialized genotypes. It could therefore provide a partial explanation for the extraordinary levels of microbial biodiversity present in many environments.

\section{Conclusions}

Our results demonstrate that toxic metabolites can have important impacts on the evolutionary processes of microbial cells. Given the ubiquity of toxic metabolites, they have implications for understanding the evolutionary histories of biological organisms. Finally, because an increased pace of evolution correlates with reduced niche breadth, the production of toxic metabolites may help to explain the enormous amount of microbial diversity in the natural environment.

\section{Methods \\ Strains and genetic manipulations}

We obtained the wild-type bacterium P. stutzeri A1501 from the Biological Resource Center of Institut Pasteur (Paris, France) and used this strain to construct all of the P. stutzeri mutant strains described in this study (Supplementary Table S3). We introduced DNA fragments containing the isopropyl- $\beta$ - $D$-thiogalactopyranosid (IPTG)-inducible $\mathrm{P}_{\text {lac }}$ promoter located immediately upstream of the egfp or echerry gene [28] into P. stutzeri A1601. These genes encode green or red fluorescent proteins, respectively. We introduced the DNA fragments using derivatives of the mini-Tn7T-LAC-Gm transposon and the pUC18T conditionally replicative delivery plasmid (Additional file 1: Table S3) as described elsewhere [29, 30]. Briefly, we constructed the derivative transposons by first purifying the pUC18T-mini-Tn7TLAC-Gm plasmid from an overnight culture of Escherichia coli DH5 $\alpha / \lambda$ pir (Additional file 1: Table S3) [31]. We next PCR amplified the egfp or echerry gene using GoTaq DNA polymerase (Promega, Madison, WI, USA) and the oligonucleotide primers listed in Supplementary Table S4. These primers contain the BamHI and KpnI restriction sites that we used to clone the PCR products into the pUC18T-mini-Tn7T-LAC-Gm plasmid. We then digested the pUC18T-mini-Tn7T-LAC-Gm plasmid and the PCR products with BamHI and KpnI (Thermo Fisher Scientific, Waltham, MA, USA) and ligated the PCR products into the pUC18T-miniTn7T-LAC-Gm plasmid. We designated the assembled derivative plasmids as pUC18T-mini-Tn7T-LACGm-egfp and pUC18T-mini-Tn7T-LAC-Gm-echerry respectively (Additional file 1: Table S3). We replicated the assembled derivative plasmids in $E$. coli DH5 $\alpha / \lambda$ pir (Additional file 1: Table S3) [31]. 
We used conjugative four-parental mating to deliver the assembled pUC18T-mini-Tn7T-LAC-Gm derivative plasmids along with the helper pUX-BF13 plasmid that expresses the transposase gene into P. stutzeri A1601 (Additional file 1: Table S1) as described elsewhere [30]. We selected $P$. stutzeri exconjugants by plating on 3-( $N$ morpholino)propanesulfonic acid (MOPS) agar plates containing $0.2 \%$ of sodium citrate and $10 \mu \mathrm{g} \mathrm{ml}^{-1}$ of gentamycin [30]. We verified plasmid segregation by testing for ampicillin sensitivity on lysogeny broth (LB) agar plates containing $100 \mu \mathrm{g} \mathrm{ml}^{-1}$ of ampicillin. We did not perform FRT excision of the gentamycin resistance marker [30] and all of the P. stutzeri mutant strains therefore retained gentamycin resistance (Additional file 1: Table S3). This was intentional to prevent contamination during the evolution experiment.

\section{Culture conditions}

We cultured all $P$. stutzeri strains under aerobic conditions in a defined asparagine-citrate synthetic medium (ACS medium) [32] with $10 \mu \mathrm{g} \mathrm{ml}^{-1}$ of gentamicin. We cultured all $P$. stutzeri strains under anaerobic conditions in dinitrogen gas $\left(\mathrm{N}_{2}\right)$-sparged ACS medium amended with $10 \mathrm{mM}$ of sodium nitrate $\left(\mathrm{NaNO}_{3}\right)$ and $10 \mu \mathrm{g} \mathrm{ml}{ }^{-1}$ of gentamycin. We reported a complete description of the methods to prepare and inoculate dinitrogen gas-sparged ACS medium elsewhere [15]. We incubated all P. stutzeri cultures at $30{ }^{\circ} \mathrm{C}$ with shaking at $220 \mathrm{rpm}$. The maximum observed nitrite concentration under these conditions was approximately $7 \mathrm{mM}$ and the $\mathrm{pH}$ did not substantially change over the course of batch growth [15]."

\section{Evolution experiment}

We experimentally evolved four populations that carry the egfp gene and four populations that carry the echerry gene for each $\mathrm{pH}$ condition ( $\mathrm{pH} 6.5$ or 7.5) for a total of 16 populations. This design controlled for any differences in the metabolic costs of carrying the egfp or echerry gene. Furthermore, we did not add IPTG to the medium during experimental evolution to avoid the metabolic cost of expressing the fluorescent proteins, and to therefore minimize the probability of selecting for loss-of-function mutations in the egfp or echerry gene over the course of the evolution experiment. We used the egfp and echerry-expressing traits to distinguish different strains during competition assays after experimental evolution (see below) and to periodically assess for cross-contamination between populations. For the latter test, we plated a small aliquot of each population onto an individual LB agar plate containing $0.1 \mathrm{mM}$ of IPTG. We never observed cross-contamination during the experiment (i.e., we never observed populations that contained both egfp and echerry-expressing clones).
For the evolution experiment, we serially transferred batch cultures containing dinitrogen gas $\left(\mathrm{N}_{2}\right)$-sparged ACS medium amended with $10 \mathrm{mM}$ of sodium nitrate $\left(\mathrm{NaNO}_{3}\right)$ as the growth-limiting substrate. We transferred each population after entering stationary phase at a dilution of 1:200 (vol:vol) (although see the exceptions below) into fresh dinitrogen gas $\left(\mathrm{N}_{2}\right)$-sparged ACS medium for a total of 700 generations. We transferred the populations evolved at $\mathrm{pH} 7.5$ (weak nitrite toxicity) every day, with some exceptions during the beginning of the evolution experiment when growth was slow or highly variable (i.e., some cultures grew slower or had long lag periods before growth was observed). We transferred the populations evolved at $\mathrm{pH} 6.5$ (strong nitrite toxicity) every fourth day during the beginning of the evolution experiment and every second day at the end of the evolution experiment. In general, we performed each transfer at a 1:200 dilution (vol:vol), with some exceptions at the beginning of the evolutionary experiments when growth was slow or highly variable. At these times, we performed each transfer at a 1:50 or a 1:100 dilution (vol:vol) and we took these exceptions into account when estimating the total number of generations. We estimated the number of generations based on the dilution, where 7.64 generations could occur from a 1:200 (vol:vol) dilution (i.e., $2^{7.64}=200$ ). This estimation is appropriate if the cultures are allowed to completely consume all of the provided nitrogen oxides prior to transfer, which was the case for our experiment. We monitored optical density at $600 \mathrm{~nm}\left(\mathrm{OD}_{600}\right)$ to verify that the cultures had entered stationary phase, and thus consumed all the nitrogen oxides, prior to transfer.

\section{Genome sequencing}

We streaked each evolved and ancestral population onto LB agar plates containing $10 \mu \mathrm{g} \mathrm{ml}^{-1}$ of gentamicin and $0.1 \mathrm{mM}$ of IPTG and picked a single colony from each population. We grew the single clones in LB overnight and extracted genomic DNA with the Wizard Genomic DNA purification kit (Promega, Madison, WI). We sequenced genomic DNA using an Illumina HiSeq 2000 sequencer (Illumina, San Diego, CA) with 100 cycles of paired-end sequencing. We performed primary data analysis, de-multiplexing, and quality control analyses of the sequencing data using FastQC (Illumina, San Diego, CA). We performed additional quality filtering of the raw reads, removed duplicate reads, and trimmed ambiguous base pairs using PRINSEQ-lite v0.20.4 [33]. Finally, we identified differences between the evolved genomes and the ancestral genome using Breseq v.0.24rc5 as described elsewhere [34]. These genetic differences included synonymous and non-synonymous point mutations, insertions, deletions, and multiplications. A complete list of mutations and their genetic targets are provided in 
Additional file 1: Table S1-2. The Quantitative Genomics Facility at ETH Zürich (Basel, Switzerland) performed all of the sequencing. The Genetic Diversity Center at ETH Zürich (Zürich, Switzerland) performed all of the bioinformatics analyses. All of the sequence reads are publically available in the European Nucleotide Archive (http://www.ebi.ac.uk/ena) under accession number PRJEB18464.

\section{Growth measurements}

We measured the time for populations to enter stationary phase by measuring $\mathrm{OD}_{600}$ over time. We plated each evolved population on LB agar plates containing $0.1 \mathrm{mM}$ of IPTG, picked ten individual colonies, inoculated the colonies into separate one-mL cultures containing aerobic ACS medium, and let them grow overnight to stationary phase. We then mixed the individual one-ml cultures from each evolved population in equal volumes, inoculated $800 \mu \mathrm{L}$ of the mixture into $20 \mathrm{~mL}$ of dinitrogen gas $\left(\mathrm{N}_{2}\right)$-sparged ACS medium amended with $10 \mathrm{mM}$ of sodium nitrate $\left(\mathrm{NaNO}_{3}\right)$, and grew the mixture to stationary phase. We used mixtures of ten individual clones from each population to maintain genetic variation of the population. We then inoculated $200 \mu \mathrm{L}$ of this stationary phase culture into $20 \mathrm{~mL}$ of fresh dinitrogen gas $\left(\mathrm{N}_{2}\right)$-sparged ACS medium amended with $10 \mathrm{mM}$ of sodium nitrate $\left(\mathrm{NaNO}_{3}\right)$ and measured $\mathrm{OD}_{600}$ over time with a Synergy plate reader (BioTek, Luzern, Switzerland) until reaching stationary phase.

\section{Competition assays}

We competed a randomly picked clone from each evolved population against the ancestral population that expresses a different fluorescent protein-encoding gene (i.e., we competed an egfp-expressing evolved clone against the echerry-expressing ancestral clone and vice versa). This allowed us to distinguish and quantify the frequencies of different strains when competed against each other. To perform the competition assays, we first plated each evolved population on LB agar plates containing $0.1 \mathrm{mM}$ of IPTG, picked a single random colony from each population, inoculated the colonies into separate one-mL cultures of aerobic ACS medium, and grew them overnight to stationary phase. We then inoculated $800 \mu \mathrm{L}$ of aerobically grown cells into $20 \mathrm{~mL}$ of dinitrogen gas $\left(\mathrm{N}_{2}\right)$-sparged ACS medium amended with $10 \mathrm{mM}$ of sodium nitrate $\left(\mathrm{NaNO}_{3}\right)$ and grew the cultures until reaching stationary phase. We finally mixed an aliquot of each evolved clone with an aliquot from the ancestral culture (50:50 or 5:95 vol:vol, depending on the experiment). We then inoculated $100 \mu \mathrm{L}$ of this mixture into $20 \mathrm{~mL}$ of fresh dinitrogen gas $\left(\mathrm{N}_{2}\right)$-sparged ACS medium amended with $10 \mathrm{mM}$ of sodium nitrate $\left(\mathrm{NaNO}_{3}\right)$ and grew the cultures until reaching stationary phase. We plated this mixture onto LB agar plates (two per competition) containing $0.1 \mathrm{mM}$ IPTG prior to incubation and after reaching stationary phase to determine the initial and final frequencies of evolved and ancestral cells. We calculated the fitness of the evolved clones relative to the ancestral clone using equation (1) where W is the relative fitness of the evolved clone, $R_{0}$ is the frequency of evolved cells before competition, $R_{1}$ is the frequency of evolved cells after competition, and $\mathrm{F}$ is the fold increase in cell numbers during the competition experiment as determined by the dilution $[35,36]$.

$$
\mathrm{W}=\frac{\log \left(\frac{\mathrm{R}_{1} \times \mathrm{F}}{\mathrm{R}_{0}}\right)}{\log \left(\frac{\left(1-\mathrm{R}_{1}\right) \times \mathrm{F}}{1-\mathrm{R}_{0}}\right)}
$$

\section{Biolog assays}

We plated each population on LB agar plates containing $0.1 \mathrm{mM}$ IPTG, picked ten individual colonies, inoculated the colonies into separate one-mL cultures of aerobic ACS medium, and let them grow overnight to stationary phase. We then mixed the individual one-ml cultures from each population in equal volumes, diluted the mixture into aerobic ACS medium lacking a carbon source (no citrate or asparagine), and pipetted the mixture into each well of a 96-well PM1 Biolog plate (Biolog, Hayward, CA, USA), where each well contained a different carbon source. We evaluated one PM1 Biolog plate per evolved population and three PM1 Biolog plates for each ancestral population (i.e., three PM1 Biolog plates for the egfp-containing strain and three PM1 Biolog plates for the echerry-containing strain). We incubated the plates at $30{ }^{\circ} \mathrm{C}$ with shaking at $220 \mathrm{rpm}$ for $24 \mathrm{~h}$ and measured the $\mathrm{OD}_{600}$ with an Eon plate reader (BioTek, Luzern, Switzerland) every second hour. We did not use a respiration indicator as is typical for Biolog analyses, and we therefore measured cell density rather than $\mathrm{cu}$ mulative respiration activity. We did this because cell density provides a more direct measure of growth than does respiration activity. We estimated growth performance for each carbon source provided in the PM1 Biolog plates by calculating the area under the curve (growth over time), which account for both growth rate and yield [22]. We normalized the data by calculating the average for the evolved clone measurements and dividing that value by the average of the ancestor measurements for each carbon source.

\section{Statistical analyses}

We used the non-parametric Wilcoxon rank-sum test to test for differences between different evolution conditions (i.e., $\mathrm{pH}$ treatments). This test is more robust to outliers than its parametric equivalent, which is particularly relevant 
to this study. Briefly, we found that one population followed a very different evolutionary trajectory than the other populations (see results section), thus reducing the power of parametric tests and questioning the validity of the normality assumption required by these tests. We tested for differences between groups using analysis of variance (ANOVA) with a subsequent Tukey's Honest Significant Difference (HSD) post hoc-test. For these analyses, we did not detect any clear outliers, and parametric analyses were therefore deemed appropriate.

\section{Additional file}

Additional file 1: Figure S1. The number of non-synonymous mutations in coding regions in the evolved clones for each experimental evolution condition. Horizontal bars and P-values indicate the outcomes from two-sample Wilcoxon rank-sum tests. A star indicates a $P$-value $<0.05$. Data are presented as Tukey box-plots. Figure S2. The relative fitness of the evolved clones calculated from competition with the ancestor at the conditions of the evolution with an inital frequency of $50 \%$ of evolved cultures. There is no significant difference in the increase in fitness between $\mathrm{pH} 7.5$ and $\mathrm{pH} 6.5$ (Wilcoxon rank sum test, $P>0.4$, $\mathrm{n}_{1}=\mathrm{n}_{2}=8$ ). Table S1. Mutations that accumulated in clones evolved at pH 7.5. Table S2. Mutations that accumulated in clones evolved at pH 6.5. Table S3. Strains and plasmids used in this study. Table S4. Oligonucleotide PCR primers used for cloning the egfp or echerry gene into the pUC18T-mini-Zn7T-LAC-Gm plasmid. (PDF $239 \mathrm{~kb}$ )

\section{Acknowledgements}

We thank Martin Ackermann for useful discussions and Lara Pfister for assistance with the genetic modifications.

\section{Funding}

This work was supported by a grant from the Swiss National Science Foundation (project number 31003A_149304). Portions of the data produced and analyzed in this manuscript were generated in collaboration with the Genetic Diversity Centre (GDC), ETH Zürich.

\section{Availability of data and materials}

All of the sequence reads analyzed for this publication are publically available in the European Nucleotide Archive (http://www.ebi.ac.uk/ena) under accession number PRJEB18464.

\section{Authors' contribution}

Both EEL and DRJ conceived the research questions and designed the experiments. EEL performed the experiments. Both EEL and DRJ wrote the manuscript. Both authors read and approved the final manuscript.

\section{Competing interests}

The authors declare that they have no competing interests.

\section{Consent for publication}

Not applicable.

\section{Ethics approval and consent to participate}

Not applicable.

\section{Author details}

'Department of Environmental Microbiology, Eawag, Überlandstrasse 133 8600 Dübendorf, Switzerland. ${ }^{2}$ Department of Environmental Systems Science, ETH Zürich, Zürich, Switzerland.
Received: 23 September 2016 Accepted: 6 February 2017

Published online: 14 February 2017

\section{References}

1. de Lorenzo V, Sekowska A, Danchin A. Chemical reactivity drives spatiotemporal organisation of bacterial metabolism. FEMS Microbiol Rev. 2015:39:96-119.

2. Ferguson SJ. Denitrification and its control. Antonie Van Leeuwenhoek. 1994;68:89-110.

3. Zumft WG. The biological role of nitric oxide in bacteria. Arch Microbiol. 1993:160:253-64

4. Vollack K, Zumft WG. Nitric oxide signaling and transcriptional control of denitrification genes in Pseudomonas stutzeri. J Bacteriol. 2001:183:2516-26.

5. Nathan CF, Hibbs JB. Role of nitric oxide synthesis in macrophage antimicrobial activity. Curr Opin Immunol. 1991:3:65-70.

6. Zumft WG. Cell biology and molecular basis of denitrification. Microbiol Mol Biol Rev. 1997;61:533-616.

7. Galhardo RS, Hastings PJ, Rosenberg SM. Mutation as a stress response and the regulation of evolvability. Crit Rev Biochem Mol Biol. 2007:42:399-435.

8. MacLean RC, Torres-Barceló C, Moxon R. Evaluating evolutionary models of stress-induced mutagenesis in bacteria. Nat Rev Genet. 2013;14:221-7.

9. Barrett RDH, M'Gonigle LK, Otto SP. The distribution of beneficial mutant effects under strong selection. Genetics. 2006;174:2071-9.

10. Barrett RDH, MacLean RC, Bell G. Mutations of intermediate effect are responsible for adaptation in evolving Pseudomonas fluorescens populations. Biol Lett. 2006:2:236-8.

11. Yan Y, Yang J, Dou Y, Chen M, Ping S, Peng J, et al. Nitrogen fixation island and rhizosphere competence traits in the genome of root-associated Pseudomonas stutzeri A1501. Proc Natl Acad Sci U S A. 2008;105:7564-9.

12. Yan Y, Ping S, Peng J, Han Y, Li L, Yang J, et al. Global transcriptional analysis of nitrogen fixation and ammonium repression in root-associated Pseudomonas stutzeri A1501. BMC Genomics. 2010;11:11.

13. Lalucat J, Bennasar A, Bosch R, García-Valdés E, Palleroni NJ. Biology of Pseudomonas stutzeri. Microbiol Mol Biol Rev. 2006;70:510-47.

14. Baumann B, Van Der Meer JR, Snozzi M, Zehnder AJB. Inhibition of denitrification activity but not of mRNA induction in Paracoccus dentrificans by nitrite at a suboptimal pH. Antonie van Leeuwenhoek. 1997;72:183-9.

15. Lilja EE, Johnson DR. Segregating metabolic processes into different microbial cells accelerates the consumption of inhibitory substrates. ISME J. 2016:10:1568-78.

16. Sijbesma WFH, Almeida JS, Reis MAM, Santos H. Uncoupling effect of nitrite during denitrification by Pseudomonas fluorescens: An in vivo 31P-NMR study. Biotechnol Bioeng. 1996;52:176-82.

17. Zhou Y, Oehmen A, Lim M, Vadivelu V, Ng WJ. The role of nitrite and free nitrous acid (FNA) in wastewater treatment plants. Water Res. 2011;45:4672-82.

18. Almeida JS, Julio SM, Reis MAM, Carrondo MIT Nitrite inhibition of denitrification by Pseudomonas fluorescens. Biotechnol Bioeng. 1995;46:194-201.

19. Goosen N, Moolenaar GF. Role of ATP hydrolysis by UvrA and UvrB during nucleotide excision repair. Res Microbiol. 2001;152:401-9.

20. Jaciuk M, Nowak E, Skowronek K, Tańska A, Nowotny M. Structure of UvrA nucleotide excision repair protein in complex with modified DNA. Nat Struct Mol Biol. 2011;18:191-7.

21. Winsor GL, Lam DKW, Fleming L, Lo R, Whiteside MD, Yu NY, et al. Pseudomonas Genome Database: Improved comparative analysis and population genomics capability for Pseudomonas genomes. Nucleic Acids Res. 2011;39:596-600.

22. Guckert JB, Carr GJ, Johnson TD, Hamm BG, Davidson DH, Kumagai Y. Community analysis by Biolog: Curve integration for statistical analysis of activated sludge microbial habitats. J Microbiol Methods. 1996;27:183-97.

23. Bjedov I, Tenaillon O, Souza V, Denamur E, Radman M. Stress-induced mutagenesis in bacteria. Science. 2003:300:1404-9.

24. Ram Y, Hadany L. The evolution of stress-induced hypermutation in asexual populations. Evolution. 2012;66:2315-28.

25. Kryazhimskiy S, Rice DP, Jerison ER, Desai MM. Microbial evolution. Global epistasis makes adaptation predictable despite sequence-level stochasticity. Science. 2014:344:1519-22.

26. MacLean RC, Buckling A. The distribution of fitness effects of beneficial mutations in Pseudomonas aeruginosa. PLoS Genet. 2009:5:e1000406.

27. Hughes BS, Cullum AJ, Bennett AF. Evolutionary adaptation to environmental pH in experimental lineages of Escherichia coli. Evolution. 2007:61:1725-34. 
28. Minoia M, Gaillard M, Reinhard F, Stojanov M, Sentchilo V, van der Meer JR. Stochasticity and bistability in horizontal transfer control of a genomic island in Pseudomonas. Proc Natl Acad Sci U S A. 2008;105:20792-7.

29. Lambertsen L, Sternberg C, Molin S. Mini-Tn7 transposons for sitespecific tagging of bacteria with fluorescent proteins. Environ Microbiol. 2004;6:726-32.

30. Choi KH, Gaynor JB, White KG, Lopez C, Bosio CM, Karkhoff-Schweizer RR, et al. A Tn7-based broad-range bacterial cloning and expression system. Nat Methods. 2005:2:443-8

31. Miller VL, Mekalanos JJ. A novel suicide vector and its use in construction of insertion mutations: Osmoregulation of outer membrane proteins and virulence determinants in Vibrio cholerae requires toxR. J Bacteriol. 1988;170:2575-83.

32. Coyle CL, Zumft WG, Kroneck PM, Korner H, Jakob W. Nitrous oxide reductase from denitrifying Pseudomonas perfectomarina. Purification and properties of a novel multicopper enzyme. Eur J Biochem. 1985;153:459-67.

33. Schmieder R, Edwards R. Quality control and preprocessing of metagenomic datasets. Bioinformatics. 2011;27:863-4.

34. Deatherage DE, Barrick JE. Identification of mutations in laboratory evolved microbes from next-generation sequencing data using breseq. Methods Mol Biol. 2014;1151:165-8.

35. Lenski RE, Rose MR, Simpson SC, Tadler SC. Long-term experimental evolution in Escherichia coli. I. Adaptation and divergence during 2,000 generations. Am Nat. 1991;138:1315.

36. Lee MC, Chou HH, Marx CJ. Asymmetric, bimodal trade-offs during adaptation of methylobacterium to distinct growth substrates. Evolution. 2009;63:2816-30

\section{Submit your next manuscript to BioMed Central and we will help you at every step:}

- We accept pre-submission inquiries

- Our selector tool helps you to find the most relevant journal

- We provide round the clock customer support

- Convenient online submission

- Thorough peer review

- Inclusion in PubMed and all major indexing services

- Maximum visibility for your research

Submit your manuscript at www.biomedcentral.com/submit 